Original article

\title{
Comparing the frequency of osteoporosis and osteopenia in chronic hepatitis B patients with and without Tenofovir treatment
}

\author{
Eskandar Hajiani ${ }^{\mathrm{a}}$, Abazar Parsi ${ }^{\mathrm{b}}$, Seyed Saeed Seyedian ${ }^{\mathrm{b}}$, Elham Rajaei ${ }^{\mathrm{c}}$, Parisa Jolodarian ${ }^{\mathrm{d}, *}$ \\ ${ }^{a}$ School of Medicine, Research Center for Infectious Diseases of Digestive System, Ahvaz Jundishapur University of Medical Sciences, Ahvaz, Iran \\ ${ }^{\mathrm{b}}$ Research Institute for Infectious Diseases of Digestive System, Ahvaz Jundishapur University of Medical Sciences, Ahvaz, Iran \\ ${ }^{\mathrm{c}}$ Department of Internal Medicine, School of Medicine, Golestan Hospital, Ahvaz Jundishapur University of Medical Sciences, Ahvaz, Iran \\ ${ }^{\mathrm{d}}$ Resident of Internal Medicine, Ahvaz Jundishapur University of Medical Sciences, Ahvaz, Iran
}

\section{A R T I C L E I N F O}

\section{Keywords:}

Chronic hepatitis B

Tenofovir

Osteoporosis

Osteopenia

Bone mineral density

\begin{abstract}
A B S T R A C T
Introduction: Osteopenia is commonly seen in people over age 50 that have lower than average bone density but do not have osteoporosis. The diagnostic difference between osteopenia and osteoporosis is the measure of bone mineral density. Tenofovir is an antiviral drug that is approved for the treatment of chronic hepatitis B and to prevent and treat Human Immunodeficiency Viruses (HIV) infection. It may cause to reduce Bone Mineral Density (BMD) in some patients.

Objective: This study aimed Comparing the Frequency of Osteoporosis and Osteopenia in Chronic Hepatitis B Patients with and without Tenofovir treatment.

Materials and methods: This study was conducted in 2018 as a cohort study on 209 patients with chronic hepatitis B, who referred to Imam Khomeini Hospital and the Gastroenterologist's Office in Ahvaz. Patients evaluated in two groups including Tenofovir group (receiving Tenofovir for more than 12 months) $(\mathrm{n}=139)$ and control group (candidates for Tenofovir and had no history of taking Tenofovir or other anti-inflammatory drugs) ( $\mathrm{n}=70$ ). BMD was evaluated in all patients by Dual-Energy X-ray Absorptiometry (DEXA) scan and compared in the two groups.

Results: The frequency percentage of patients with osteopenia and osteoporosis in the Tenofovir group (60.43\%) was significantly more than that of the control group $(21.43 \%)(\mathrm{P}<0.05)$. Among Tenofovir group, 51.02\% of males and $42.22 \%$ of females had osteopenia. Also, $14.28 \%$ of males and $15.56 \%$ of females had osteoporosis. In the patients who had consumed more time, incidence of osteoporosis and osteopenia were reported more often $(\mathrm{P}>0.05$ ). Among patients with osteopenia, $85.71 \%$ of the patients in the hip region and $44.44 \%$ of them in the lumber area had decreased bone density. Also, osteoporosis was more reported in hip (90.48\%), lumbar $(90.48 \%)$ and femoral neck $(80.95 \%)$ respectively.

Conclusion: A significant reduction in bone density was observed in patients with chronic hepatitis B receiving Tenofovir for more than 12 months in comparison with the control group. Also, the patients who had been taking Tenofovir in longer time, osteoporosis and osteopenia were more reported.
\end{abstract}

\section{Introduction}

Hepatitis B virus infection is a serious health problem in the world, ${ }^{1-3}$ with clinical manifestations of both acute and chronic conditions. During the acute phase, the form of manifestation is from nonicteric hepatitis to icteric hepatitis and in some patients is fulminant hepatitis. ${ }^{4-7}$ During the chronic phase, the form of manifestation is from asymptomatic carriers to chronic hepatitis, cirrhosis and hepatocellular carcinoma. ${ }^{8}$ Extrahepatic manifestations can occur simultaneously with acute and chronic infection. ${ }^{9-12}$ The third generation of analog nucleotides (Tenofovir and Entecavir) is currently the first line treatment for chronic hepatitis B. Tenofovir is an acyclic nucleotide analogs adenosine monophosphate that has been shown to be effective in treating chronic hepatitis $\mathrm{B}$ and has been proposed by all guidelines. ${ }^{13,14}$ Tenofovir, as the first line of antiviral therapy, has the ability to reverse liver fibrosis and is an alternative drug in case of drug resistance. ${ }^{15-18}$ The main side effects associated with Tenofovir include renal failure, renal tubular impairment, and decreased bone density. ${ }^{19-25}$ Reductions in bone density are associated with an increase in phosphate excretion from the kidney, although this effect is mild but

\footnotetext{
* Corresponding author.

E-mail address: parisajolodarian@yahoo.com (P. Jolodarian).
} 
more evident in the varieties, therefore, Tenofovir intakes of less than 12 years should be avoided as much as possible. Possible mechanisms include renal tubular dysfunction, which leads to hypophosphatemia, and recent reports have been made on changes in osteoblasts gene expression and their function. ${ }^{19,26-29}$ Data on the effect of treatment with Tenofovir on bone mineral density (BMD) in patients with chronic hepatitis $\mathrm{B}$ infection are incomplete. ${ }^{28}$ Tenofovir is a first and effective choice for the treatment of chronic hepatitis B, but there is little research on the Tenofovir effect on osteopenia and osteoporosis. The aim of this study was to evaluate the incidence of osteopenia and osteoporosis in patients with chronic hepatitis B treated with Tenofovir, so that if Tenofovir is shown to be effective in the development of osteoporosis in patients who are candidates for receiving this drug in prophylaxis form, a drug for preventing osteoporosis, such as calcium D or bisphosphonate, should be considered.

\section{Method}

After approving the research at the ethics committee of the Jundishapur University of Ahvaz (IRI.AJUMS.REC.1397.483). This cohort study was conducted during 2018 at Imam teaching hospital of Ahvaz (a tertiary-care hospital) with 900 beds approximately, in the southwest of Iran on 209 patients with chronic hepatitis B, who referred to the gastroenterology clinic of Imam Khomeini Hospital and the Gastroenterologist's Office in Ahvaz. ${ }^{30}$ Ahvaz megacity Located in south west of Iran. It's the capital city of Khuzestan province, with an area of 140 square kilometers. ${ }^{31-33}$ Based on laboratory findings and expert confirmation, the diagnosis of chronic hepatitis B was confirmed in these patients who were treated with Tenofovir for more than 12 months. Patients with chronic hepatitis B over the age of 18, who were candidates for Tenofovir and had no history of taking Tenofovir or other anti-inflammatory drugs with effect on bone density, were considered as the control group. Exclusion criteria included the use of drugs that affect osteoporosis and osteopenia (such as steroids, etc.), a history of osteoporosis, or osteopenia, or a related fracture, the presence of concomitant illness affecting osteoporosis and osteopenia (such as chronic kidney disease, liver cirrhosis, rheumatologic disease, RA, corticosteroid, etc.) and hepatitis D and C and Human Immunodeficiency Viruses (HIV) infection. After explaining the goals of the study and the way of conducting the study, written consent was obtained from all patients. The sampling method was judgmental or purposive sampling. Patients' information was collected through their records at the physician's office and clinic of Imam Khomeini Hospital in Ahvaz and they were contacted for attendance at the clinic and performing the examinations. In order to compare the Bone Mineral Density (BMD) of the patients in both control and Tenofovir groups, a single device was used (in accordance with the bone densitometry center located at Ahvaz Golestan Hospital). The Dual-Energy X-ray Absorptiometry (DEXA) scan was analyzed by a physician and bone densitometry test was reported in three modes: $\mathrm{gr} / \mathrm{cm},{ }^{3}$ Z-score (defined as a standard deviation above or below of a person of the same age, gender, and age) and T-score (defined as the standard deviation above or below the average of a 30-year-old person of the same ethnicity and sex with the patient). According to the definition by WHO, in $\mathrm{T}$-score more than -1 , densitometry of bone is considered normal. Tscore of 1 - to -2.5 is considered as osteopenia and T-score of less than 2.5 is considered osteoporosis. The obtained data was keyed into the computer software Statistical Package for Social Sciences (SPSS) for windows version 22.0. Qualitative variables are reported as percentages, and quantitative variables are reported as the mean \pm standard deviation. A paired $t$-test and Wilcoxon's signed rank test were used to compare mean of quantitative variables. The relation between study variables was assessed using Pearson's correlation coefficient. P $<0.05$ was considered as significant level.
Table 1

Demographic data of patients in the two groups.

\begin{tabular}{lllll}
\hline Variables & & TDF $(n=139)$ & Control $(\mathrm{n}=70)$ & P-Value \\
\hline \multirow{2}{*}{ Gender } & Female & $90(64.75 \%)$ & $26(37.14 \%)$ & $<0.0001^{*}$ \\
& Male & $49(35.25 \%)$ & $44(62.86 \%)$ & \\
Age (year) & & $45.94 \pm 10.03$ & $41.73 \pm 10.48$ & 0.078 \\
\hline
\end{tabular}

\section{Results}

In this study, patients with chronic hepatitis B receiving Tenofovir ( $\mathrm{n}=139$ ) with chronic hepatitis B patients who did not receive Tenofovir $(n=70)$ aged 19-65 years were studied. The mean age of patients in both groups was not significantly different $(P>0.05)$. In the Tenofovir group, the number of female patients was higher than that of male patients, but in the control group, the number of male patients was higher and the two groups had statistically significant differences in terms of gender distribution $(P<0.05)$ (Table 1$)$.

The presence of chronic kidney disease in patients of both groups was one of the outcomes of the study and the study population at the start of the study was normal in this regard. In patients under evaluation, although the mean creatinine level in Tenofovir recipients was higher than that in the control group, the difference was not statistically significant, and both groups were within the normal range $(\mathrm{P}>0.05)$.

The distribution of the patients in both groups was significantly different based on the results of bone densitometry test so that the percentage of patients with osteopenia and osteoporosis in the Tenofovir group was significantly more than that of the control group $(\mathrm{P}<0.05)$ (Table 2).

Among Tenofovir recipients, $51.02 \%$ of the patients were male and $42.22 \%$ of the patients had osteopenia. Also, $14.28 \%$ of the male patients and $15.56 \%$ of the women patients had osteoporosis. The results showed that the prevalence was almost equal to the reduction in bone density in both genders. The percentage of patients with osteoporosis and osteopenia was calculated based on the frequency of patients and their gender in the same gender in an intragroup way. Among the patients with osteopenia and osteoporosis, the most involved part was the hip (Table 3).

There was a significant relationship between the mean duration of taking Tenofovir in patients using this medicine with osteoporosis and osteopenia so that in patients who took more of this medicine, bone loss, osteoporosis and osteopenia were reported more often $(\mathrm{P}<0.05)$ (Table 4).

In female patients, bone loss decreased significantly with TDF compared to male patients $(\mathrm{P}<0.05)$. There was no significant relationship between the mean duration of using this drug with age and osteoporosis and osteopenia. However, in the higher age group (more than 50 years), decreased bone density decreased after a shorter time, but this time was not significantly different with the time obtained for younger subjects $(\mathrm{P}>0.05)$ (Table 5$)$.

\section{Discussion}

In the present study, the distribution of patients in both groups

Table 2

Comparison of osteoporosis and osteopenia in patients in the two groups.

\begin{tabular}{llll}
\hline BMD & TDF $(\mathrm{n}=139)$ & Control $(\mathrm{n}=70)$ & P-value \\
\hline Normal & $55(39.57 \%)$ & $55(78.57 \%)$ & $<0.0001^{*}$ \\
Osteopenia & $63(45.32 \%)$ & $12(17.14 \%)$ & \\
Osteoporosis & $21(15.11 \%)$ & $3(4.29 \%)$ & \\
\hline
\end{tabular}

Data are expressed as mean \pm SD.

The statistical test used was the Chi-squared-test.

${ }^{*} \mathrm{P}<0.05$ was considered as significance level. 
Table 3

Gender relationship with osteoporosis and osteopenia in patients receiving Tenofovir.

\begin{tabular}{llll}
\hline Variables & & Osteopenia $(\mathrm{n}=63)$ & Osteoporosis ( $\mathrm{n}=21)$ \\
\hline \multirow{2}{*}{ Gender (N, \%) } & Female & $38(42.22 \%)$ & $14(15.56 \%)$ \\
& Male & $25(51.02 \%)$ & $7(14.28 \%)$ \\
Affected Area & Lumbar spine & $28(44.44 \%)$ & $19(90.48 \%)$ \\
& Hip & $54(85.71 \%)$ & $19(90.48 \%)$ \\
& Femoral Neck & 0 & $17(80.95 \%)$ \\
\hline
\end{tabular}

Table 4

The relationship between Tenofovir intake and incidence of osteoporosis and osteopenia.

\begin{tabular}{llc}
\hline BMD & Duration of consumption & P-value \\
\hline Normal & $12.73 \pm 0.849$ & $0.012^{*}$ \\
Osteopenia & $16.25 \pm 2.31$ & \\
Osteoporosis & $18.90 \pm 3.08$ & \\
\hline
\end{tabular}

Data are expressed as mean $\pm \mathrm{SD}$.

The statistical test used was the ANOVA-test.

$* \mathrm{P}<0.05$ was considered as significance level.

Table 5

Correlation between incidence of osteoporosis and osteopenia with age and gender in Tenofovir group.

\begin{tabular}{llll}
\hline BMD & & Duration of consumption & P-value \\
\hline Osteopenia & Female & $14.89 \pm 1.87$ & $<0.0001^{*}$ \\
& Male & $18.32 \pm 1.03$ & \\
Osteoporosis & Female & $17.43 \pm 2.07$ & $<0.0001^{*}$ \\
& Male & $21.86 \pm 2.73$ & 0.380 \\
Osteopenia & $<50$ yrs & $16.00 \pm 2.09$ & \\
& $\geq 50$ yrs & $16.52 \pm 2.53$ & 0.573 \\
Osteoporosis & $<50$ yrs & $19.13 \pm 3.40$ & \\
& $\geq 50$ yrs & $18.20 \pm 1.92$ & \\
\hline
\end{tabular}

Data are expressed as mean \pm SD.

The statistical test used was the $t$-test.

${ }^{*} \mathrm{P}<0.05$ was considered as significance level.

based on bone densitometry test results was significantly different, so that the percentage of patients with osteopenia and osteoporosis in the tenofovir group $(60.43 \%)$ was significantly more than that of the control group (21.43\%) $(\mathrm{P}<0.05)$. The results of the Upkar.S.Gill (2014) study showed a reduction in bone density in chronic hepatitis B patients receiving Tenofovir for more than 12 months versus the control group. ${ }^{28}$ The results of Scott Fong et al. (2014) in patients with chronic hepatitis B treated only with Tenofovir, compared with Tenofovir with emtricitabine for 96 weeks, showed a slight reduction of less than $2 \%$ in the densitometric mean of hip and vertebral bone in both groups. ${ }^{19}$ In a study by Murray et al. (2012) that examined the safety and efficacy of TDF in adolescents with chronic TDV-treated hepatitis B, in none of the patients, reduced bone density in the spine at $6 \%$ of endpoint security was not reported within 72 weeks. ${ }^{29}$ In this study, among Tenofovir recipients, $51.02 \%$ of males and $42.22 \%$ of females had osteopenia. Also, $14.28 \%$ of males and $15.56 \%$ of females had osteoporosis. The results showed that the incidence of bone density reduction in both sexes is approximately equal. There was a significant relationship between the mean duration of taking Tenofovir in patients using this drug with osteoporosis and osteopenia so that in patients who had consumed more of this drug, osteoporosis, and osteopenia were reported more often $(P>0.05)$. In female patients, bone density reduction occurred in a significantly shorter time after using TDF than in male patients, and administration of BMD enhancers and drugs preventing its reduction, such as calcium and vitamin $\mathrm{D}$, as well as screening the bone density condition according to the scheduled timing seems more essential in these patients. In the study of Upkar.S.Gill (2014), there was no significant correlation between the duration of Tenofovir use and BMD changes. ${ }^{28}$ There was no significant relationship between median duration of drug use and age and osteoporosis and osteopenia. However, in the higher age group (more than 50 years), decreased bone density was less than the medication consumption, but this time with the amount there was no significant difference in younger subjects $(\mathrm{P}<0.05)$.

Among patients with osteopenia, $85.71 \%$ of the patients in the hip region and $44.44 \%$ of them in the lumbric area had decreased bone density. Also, in patients with osteoporosis, $90.48 \%$ of patients in the hip region, $90.48 \%$ of them in the lumbar region, and $80.95 \%$ of them in the femoral neck area had decreased bone density. The results of the study by Wong Gl et al. (2015) in a three-year analysis showed that treatment with HA had a slightly higher risk of hip fracture in chronic hepatitis B patients receiving a nucleotide analog (such as Tenofovir) compared to those receiving a nucleoside analog. ${ }^{25}$ In the study of Upkar.S.Gill (2014), BMD was significantly reduced in hepatitis B patients receiving Tenofovir $(\mathrm{p}=0.02)$. In the Tenofovir recipient group, osteopenia was reported in $37 \%$ of patients in the lumbar, $40 \%$ in the hip and $35 \%$ in the femoral neck. ${ }^{28}$ In a study by Fung et al. (2017) that looked at the long-term effects of taking Tenofovir disoproxil fumarate (TDF) alone or in combination with emtricitabine (FTC) over 240 weeks, overall changes in BMD in the spine were $-0.98 \%$ and $-2.54 \%$ in the pelvis. ${ }^{27}$ Although the mean creatinine level in the Tenofovir group was higher than the control group, the difference was not statistically significant $(\mathrm{P}<0.05)$. The results of Wong Gl et al. (2015), in a three-year analysis, showed that treatment with NA does not increase the risk of kidney events in general. ${ }^{25}$

The main limitation of the current study appears to be a relatively small sample size.

\section{Conclusion}

Based on the results of this study, the percentage of patients with osteopenia and osteoporosis in the Tenofovir group was significantly more than that of the control group. Also, a significant reduction in bone density was observed in patients with chronic hepatitis B receiving Tenofovir for more than 12 months in comparison with the control group. The prevalence of this disorder in both sexes was almost equal. Also, there was a significant association between the mean time of using Tenofovir in patients receiving this drug with osteoporosis and osteopenia, so that in patients who had been taking this drug longer, osteoporosis and osteopenia were more commonly reported.

\section{Declaration of competing interest}

No potential conflict of interest relevant to this article was reported.

\section{Acknowledgment}

This research was supported by grants (RDC-9706) from the Vice Chancellor of Research and development, Ahvaz Jundishapur University of Medical Sciences (Iran). This study is residency thesis of Dr. Parisa Jolodarian.

\section{References}

1. Maynard J. Hepatitis B: global importance and need for control. Vaccine 1990;8:S18-S20.

2. Ott J, Stevens G, Groeger J, Wiersma S. Global epidemiology of hepatitis B virus infection: new estimates of age-specific HBsAg seroprevalence and endemicity. Vaccine. 2012;30:2212-2219.

3. Schweitzer A, Horn J, Mikolajczyk RT, Krause G, Ott JJ. Estimations of worldwide prevalence of chronic hepatitis B virus infection: a systematic review of data published between 1965 and 2013. The Lancet. 2015;386:1546-1555.

4. Garfein RS, Bower WA, Loney CM, et al. Factors associated with fulminant liver failure during an outbreak among injection drug users with acute hepatitis B. Hepatology. 2004;40:865-873. 
5. Liaw Y-F, Tsai S-L, Sheen I-S, et al. Clinical and virological course of chronic hepatitis $\mathrm{B}$ virus infection with hepatitis C and D virus markers. Am J Gastroenterol. 1998;93:354-359.

6. Sato S, Suzuki K, Akahane Y, et al. Hepatitis B virus strains with mutations in the core promoter in patients with fulminant hepatitis. Ann Intern Med. 1995;122:241-248.

7. Wright TL, Mamish D, Combs C, et al. Hepatitis B virus and apparent fulminant nonA, non-B hepatitis. The Lancet. 1992;339:952-955.

8. Lok AS, Lai CL. $\alpha$-fetoprotein monitoring in Chinese patients with chronic hepatitis B virus infection: role in the early detection of hepatocellular carcinoma. Hepatology. 1989;9:110-115

9. CY L. Clinical features and natural course of HIV-related glomerulopathy in children. Kidney Suppl. 1991;35:35-46.

10. Guillevin L, Lhote F, Cohen P, et al. Polyarteritis nodosa related to hepatitis B virus. A prospective study with long-term observation of 41 patients. Medicine. 1995; 74:238-253.

11. Johnson RJ, Couser WG. Hepatitis B infection and renal disease: clinical, immunopathogenetic and therapeutic considerations. Kidney Int. 1990;37:663-676.

12. LAI KN, LAI FM-M, CHAN KW, CHOW CB, TONG KL, VALLANCE-OWEN J. The clinico-pathologic features of hepatitis B virus-associated glomerulonephritis. QJM: Int J Med. 1987;63:323-333.

13. Liver EAFTSOT. EASL clinical practice guidelines: management of chronic hepatitis B virus infection. $J$ Hepatol. 2012;57:167-185.

14. Lok AS, McMahon BJ. Chronic hepatitis B: update 2009. Hepatology. 2009;50:661-662

15. Buti M, Gane E, Seto WK, et al. Tenofovir alafenamide versus tenofovir disoproxil fumarate for the treatment of patients with $\mathrm{HBeAg}$-negative chronic hepatitis B virus infection: a randomised, double-blind, phase 3, non-inferiority trial. Lancet Gastroenterol Hepatol. 2016;1:196-206.

16. Marcellin P, Gane E, Buti M, et al. Regression of cirrhosis during treatment with tenofovir disoproxil fumarate for chronic hepatitis B: a 5-year open-label follow-up study. The Lancet. 2013;381:468-475.

17. Tourret J, Deray G, Isnard-Bagnis C. Tenofovir effect on the kidneys of HIV-infected patients: a double-edged sword? J Am Soc Nephrol. 2013;24:1519-1527.

18. Wong GLH, Chan HLY, Mak CWH, et al. Entecavir treatment reduces hepatic events and deaths in chronic hepatitis B patients with liver cirrhosis. Hepatology. 2013;58:1537-1547.

19. Fung S, Kwan P, Fabri M, et al. Randomized comparison of tenofovir disoproxil fumarate vs emtricitabine and tenofovir disoproxil fumarate in patients with lamivudine-resistant chronic hepatitis B. Gastroenterology. 2014;146:980-988 e981.

20. Gara N, Zhao X, Collins M, et al. Renal tubular dysfunction during long-term adefovir or tenofovir therapy in chronic hepatitis B. Aliment Pharmacol Ther. 2012;35:1317-1325.

21. Gupta SK. Tenofovir-associated Fanconi syndrome: review of the FDA adverse event reporting system. AIDS Patient Care STDS. 2008;22:99-103.

22. Lampertico P, Chan H, Janssen H, Strasser S, Schindler R, Berg T. long-term safety of nucleoside and nucleotide analogues in HBV-monoinfected patients. Aliment Pharmacol Ther. 2016;44:16-34.

23. Pipili C, Cholongitas E, Papatheodoridis G. Nucleos (t) ide analogues in patients with chronic hepatitis B virus infection and chronic kidney disease. Aliment Pharmacol Ther. 2014;39:35-46.

24. Verhelst D, Monge M, Meynard J-L, et al. Fanconi syndrome and renal failure induced by tenofovir: a first case report. Am J Kidney Dis. 2002;40:1331-1333.

25. Wong GLH, Tse YK, Wong VWS, Yip TCF, Tsoi KKF, Chan HLY. Long-term safety of oral nucleos ( $\mathrm{t}$ ) ide analogs for patients with chronic hepatitis B: a cohort study of 53,500 subjects. Hepatology. 2015;62:684-693.

26. Fontana RJ. Side effects of long-term oral antiviral therapy for hepatitis B. Hepatology. 2009;49:S185-S195

27. Fung S, Kwan P, Fabri M, et al. Tenofovir disoproxil fumarate (TDF) vs. emtricitabine (FTC)/TDF in lamivudine resistant hepatitis B: a 5-year randomised study. J Hepatol. 2017;66:11-18.

28. Gill US, Zissimopoulos A, Al-Shamma S, et al. Assessment of bone mineral density in tenofovir-treated patients with chronic hepatitis B: can the fracture risk assessment tool identify those at greatest risk? J Infect Dis. 2014;211:374-382.

29. Murray KF, Szenborn L, Wysocki J, et al. Randomized, placebo-controlled trial of tenofovir disoproxil fumarate in adolescents with chronic hepatitis B. Hepatology. 2012;56:2018-2026.

30. Masjedizade A, Mohammadi MJ, Yazdankhah S, Roumi A, Shahriari A, Geravandi S The effect of reducing the incidence of gastrointestinal complications in patients treated with aspirin, referred to Imam Hospital, of Ahvaz, Iran. Data Brief. 2017; 15:478-482

31. Nashibi R, Afzalzadeh S, Mohammadi MJ, Yari AR, Yousefi F. Epidemiology and treatment outcome of mucormycosis in Khuzestan, Southwest of Iran. Arch Clin Infect Dis. 2017;12:e37221

32. Dastoorpoor M, Sekhavatpour Z, Masoumi K, et al. Air pollution and hospital admissions for cardiovascular diseases in Ahvaz, Iran. Sci Total Environ. 2019;652:1318-1330.

33. Soltani F, Ghomeishi A, Mohammadi MJ, et al. Association of toxic microbial and chemical water quality of hemodialysis instruments during 2016. Fresenius Environ Bull. 2017;26:5357-5362. 